\section{Should Tuberculin Skin Test Be Positive to Give Latent Tuberculosis Treatment Before Tumor Necrosis Factor- $\alpha$ Inhibitors in Selected Patients in Developing Countries?}

\section{To the Editor:}

We read with interest the report by Malaviya, et al about tuberculosis (TB) and inflammatory rheumatic disease (IRD) patients receiving tumor necrosis factor- $\alpha$ (TNF- $\alpha$ ) inhibitor agents ${ }^{1}$. Mexico also has a "high TB burden," with estimated prevalence of at least 23 per $100,000^{2}$. The tuberculin skin test (TST) has low sensitivity, including in high-risk population groups such as men and patients older than 50 years, in addition to those who have disseminated $\mathrm{TB}^{3,4}$. In immunosuppressed patients (such as those with human immunodeficiency virus), tuberculin anergy is reported to be $>40 \%$, particularly in those with poor body mass index and/or severe lymphopenia ${ }^{5}$. This could also be true about other immunodeficient persons, including patients with rheumatic disease who are taking disease-modifying antirheumatic drugs (DMARD) and corticosteroids $\mathrm{s}^{6,7}$.

One of 22 patients reported by Malaviya, et al who tested negative for TB (TST, Quanti-FERON-TB Gold, standard chest radiograph, and computed tomography scan) developed TB 8 weeks after 1 dose of TNF- $\alpha$ inhibitor agents ${ }^{1}$. We have 5 patients with IRD with TST-negative active TB; 1 of them had systemic lupus erythematosus (SLE) treated with infliximab because of refractory polyarthritis. That patient had acute respiratory failure secondary to disseminated fulminating TB, identified in necropsy 2 weeks after death to have received the first administration of infliximab (previous normal chest radiograph). Only 1 of these patients with IRD was not treated with DMARD and received prednisone $>10 \mathrm{mg}$ (Table 1).

To see whether there were differences among our cases and patients without IRD, we evaluated 54 patients without IRD and with active TB (excluding other immune-suppression causes). The average age was 39.4 years; $51.9 \%$ were female and $29.6 \%$ TST- negative; most of them had pulmonary TB (61\%). We did not find TST differences between patients with pulmonary disease $(70 \%, 23 / 33)$ and those with extrapulmonary disease $(71.4 \%, 15 / 21 ; p>0.05)$. Moreover, our healthy population has a high frequency of positive TST (> $10 \mathrm{~mm}$ ). We found this in $65 \%$ of 650 first-year medical students (mean age $18 \mathrm{yrs}$ ), who had not started clinical practice and did not have any potential contact with TB patients. But we cannot explain why $<20 \%$ of our IRD patients have positive TST.

It is evident that our patients have a high risk of developing active TB independent of TST results. It is interesting that the treatment with TNF- $\alpha$ inhibitor agents does not decrease the sensitivity of TST. In addition, we have found that $10 \%$ of patients under biologic therapy (non-TNF- $\alpha$ inhibitor agents) who previously were TST-negative can change to positive. It has also been found that patients taking TNF- $\alpha$ inhibitor agents maintained or increased their TST positivity ${ }^{8}$. Developed countries have reported patients with previous negative TST being diagnosed with TB after taking TNF- $\alpha$ inhibitor agents and normal chest radiograph ${ }^{9}$. These considerations are important because we recognize that the TST has low sensitivity for diagnosis of latent TB. Malaviya, et al reported in India an incidence of $18.2 \%$ of TB disease flare in patients treated with TNF- $\alpha$ inhibitor agents with a "standard" screening strategy for latent TB, and $4.5 \%$ with the "modified" TB screening strategy ${ }^{1}$.
For developing countries where latent TB has a high prevalence, and despite negative screening tests for $\mathrm{TB}$, we propose that before starting TNF- $\alpha$ inhibitor treatment, patients should receive prophylactic therapy or treatment for latent TB. TB disease flares could be brought close to zero, or at least near $1 \%$ as quoted from randomized controlled trials of TNF- $\alpha$ inhibitor agents ${ }^{10}$

In Mexico, as in other developing countries having high TB prevalence, it could be reasonable to receive therapy for latent TB before taking TNF- $\alpha$ inhibitor agents, independent of the TST results. We do not know the maintenance time for TB therapy, because we do not have evidence that after the recommended 9 months of therapy, the TB risk could be modified. These considerations are expressed because although we had only 5 patients with IRD who developed active TB, one of them under TNF- $\alpha$ inhibitor therapy, the diagnosis of active TB was made in most of our patients with invasive methods.

\section{CARLOS ABUD-MENDOZA, MD; MARCO ULISES}

MARTÍNEZ-MARTÍNEZ, MD, Regional Unit of Rheumatology and Osteoporosis Hospital Central, Faculty of Medicine, Universidad Autonoma de San Luis Potosi; JOSÉ DE JESÚS MACÍAS-MENDOZA, MD; MARTÍN MAGAÑA-AQUINO, MD, Faculty of Medicine, San Luis Potosi, Mexico. Address correspondence to Dr. Abud-Mendoza; E-mail: c_abud@hotmail.com

\section{REFERENCES}

1. Malaviya AN, Kapoor S, Garg S, Rawat R, Shankar S, Nagpal S, et al. Preventing tuberculosis flare in patients with inflammatory rheumatic diseases receiving tumor necrosis factor- $\alpha$ inhibitors in India - an audit report. J Rheumatol 2009;36:1414-20.

2. WHO-Global tuberculosis database -2007 . Available from http://apps.who.int/globalatlas/dataQuery/default.asp. Accessed June 7, 2009.

3. Holden M, Dubin MR, Diamond PH. Frequency of negative intermediate-strength tuberculin sensitivity in patients with active tuberculosis. N Engl J Med 1971;285:1506-9.

4. Nash DR, Douglass JE. Anergy in active pulmonary tuberculosis. A comparison between positive and negative reactors and an evaluation of $5 \mathrm{TU}$ and $250 \mathrm{TU}$ skin test doses. Chest 1980;77:32-7

5. Johnson JL, Nyole S, Okwera A, Whalen CC, Nsubuga P, Pekovic $\mathrm{V}$, et al. Instability of tuberculin and Candida skin test reactivity in HIV-infected Ugandans. The Uganda-Case Western Reserve University Research Collaboration. Am J Respir Crit Care Med 1998;158:1790-6.

6. Smirnoff M, Patt C, Seckler B, Adler JJ. Tuberculin and anergy skin testing of patients receiving long-term hemodialysis. Chest 1998;113:25-7.

7. Chan YC, Yosipovitch G. Suggested guidelines for screening and management of tuberculosis in patients taking oral glucocorticoids - an important but often neglected issue. J Am Acad Dermatol 2003;49:91-5

8. Fuchs I, Avnon L, Freud T, Abu-Shakra M. Repeated tuberculin

Table 1. Data of 5 patients with active TB-IRD.

\begin{tabular}{lcccccc}
\hline Case & Sex & Age, yrs & Disease & Time of Diagnosis & Treatment & Diagnosis of TB \\
\hline 1 & M & 22 & AS & 4 yrs & PDN, MTX & Bacilloscopy \\
2 & F & 29 & SLE & 5 mo & PDN, MTX, AZA & Biopsy \\
3 & F & 34 & SLE & 0 yrs & None & Biopsy \\
4 & F & 62 & RA & 4 yrs & PDN, MTX & Biopsy \\
5 & F & 20 & SLE & $1 \mathrm{yr}$ & PDN, MTX, infliximab & Autopsy \\
\hline
\end{tabular}

TB: tuberculosis; IRD: inflammatory rheumatic disease; AS: ankylosing spondylitis; SLE: systemic lupus erythematosus; RA: rheumatoid arthritis; PDN: prednisone; MTX: methotrexate; AZA: azathioprine. 
skin testing following therapy with TNF-alpha inhibitors. Clin Rheumatol 2009;28:167-72.

9. Arend SM, Leyten EM, Franken WP, Huisman EM, van Dissel JT. A patient with de novo tuberculosis during anti-tumor necrosis factor-alpha therapy illustrating diagnostic pitfalls and paradoxical response to treatment. Clin Infect Dis 2007;45:1470-5.
10. Keane J, Bresnihan B. Tuberculosis reactivation during immunosuppressive therapy in rheumatic diseases: diagnostic and therapeutic strategies. Curr Opin Rheumatol 2008;20:443-9.

J Rheumatol 2010; 37:3; doi:10.3899/jrheum.090672 